\title{
One-Pot Green Method for the Synthesis of Oxazine Derivatives Under Aqueous Medium
}

\author{
Santosh R. Kshirsagar, Ashok S. Pise, Sagar I. Shinde, Sandip P. Gondake, Valmik S. Kapase \\ Department of Chemistry, Dada Patil Mahavidyalaya, Karjat, Ahmednagar, Maharashtra, India \\ *Corresponding Author: Email: : sksantosh84@gmail.com
}

\author{
Article Info \\ Volume 7, Issue 4 \\ Page Number: 264-270 \\ Publication Issue : \\ July-August-2020
}

\section{Article History}

Accepted : 15 Aug 2020

Published : 25 Aug 2020

\section{ABSTRACT}

A simple, convenient, and environmentally friendly method for the synthesis of 1,3-oxazine derivatives has been developed under aqueous conditions. The reaction proceeds via one pot multicomponent condensation of $\alpha$ or $\beta$ naphthol, aromatic aniline and formaldehyde using polyphosphoric acid as green catalyst. The current protocols are simple, requires less reaction time and provides high yields. Non-polluting synthetic procedures are used to avoid harmful effects of organic solvents on the environment.

Keywords : 1, 3-oxazine derivatives, One-pot multicomponent condensation, Polyphosphoric acid, Aqueous medium, Green method.

\section{INTRODUCTION}

The environmentally friendly and economically inexpensive synthetic procedures have been developed toreduce the harmful effect of organic solvent on the environment. The main objective of this work was to avoid the use of harmful solvents and catalysts. Nowadays, synthetic chemists are facing the major challengesin chemistry due to the limited green options for the synthesis of heterocycles. Synthesis of biologically active compounds containing nitrogen and oxygen in a ring from readilyavailable reagents is the main objective of organic synthesis[1]. The multi-component condensation reactions have attracted attention during the last few years to increase the number of organic transformations because it is a rapid and efficient method for the formation of molecules in a single-step[2].
Multicomponent reactions(MCRs) are useful for C-C and C-heteroatom bond formations and also helpful to synthesize small molecules with structural diversity[3].The MCRs combined with convergence and atom economy, this type of reaction has many applications in organic and Medicinal Chemistry[4].The design and synthesis of new heterocycles which has medicinal and biological activity in cost and time effective manner is a goal[5$11]$.

The heterocyclic compounds are found in nature and play an important role in human life.Out of a large number of heterocyclic compounds, 1,3-oxazine hasspecial interest because it is useful for a variety of functional group interconversion. In recent years, these1, 3-oxazineshave been tested for various biological properties such as analgesic, antifungal, 
anticonvulsant [12-14], antitubercular[15], antihypertensive [16], antiulcer [17], antithrombotic [18], antibacterial[19], and high activity against HIV1 mutant strain[20]. Also, the napthoxazinederivatives used for the treatment of Parkinson's diseases[21]. The tatumer character of 1,3 oxazinesoffer a large number of synthetic possibilities[15]. The 1,3 oxazines are photochromic compounds [22] as well as it has thiolfunction(-SH enzymes) essential for enzyme activity leads to possess biological and therapeutic properties[23-30]. It is used as an intermediate in the synthesis of $\mathrm{N}$ substituted amino alcohols[31]. Due to chemical and biological interest, the synthesis of various substituted and unsubstituted 1,3 oxazineshave been done.

Owing to the usefulness and importance of the 1,3oxazines, many organic researchers previously reported the mannich type condensation of primary amine, phenol or naphthol with formaldehyde using organic solvents[32] and alkaline medium[33].Similarly, mild, solvent-free, water and ionic method are also reported[34-36]. The1,3oxazinederivatives were formed from the threecomponent cyclocondensation of primary aliphatic and cyclic amines with formaldehyde and substituted phenol [37]. Overall, the yield of productswas depending on the amine, temperature, nature, and substituent of phenol.Different methods have been reported so far for the synthesis of 1,3 oxazine[38-40] but only few methods used the MCRs approaches [41]. Recently, the 1,3-oxazine derivatives were synthesized by one-pot multicomponent condensation of naphthol, an aniline and formaldehyde usingthiamine hydrochloride catalyst [42]. Thesereactions required harsh reaction conditions, produces lower product yields, requires longer reaction time withtediousworkup procedure. The attention is paid to search convenient and efficient methods based onthe green approach.
The multicomponent reaction strategy is to combine the economic aspect with the environmental one. The synthesis is carried out in one-step without isolating intermediate reduce time, money, energy, and raw materials [43]. Nowadays chemists focus on green and clean approach by using nontoxic solvent, reagent, and catalyst [44].The green synthetic methods used in aqueous medium. Water is more abundant and the cheapest and environmentally friendly solventand has unique reactivity and selectivity [45]. The polyphosphoric acid is the most commonly used catalyst for dehydration and cyclization [46]. In our knowledge, there are no reports on the synthesis of 1,3-oxazines by using polyphosphoric acid as catalyst.This novel method has been designed for multicomponent synthesis using water as a green solvent .In the present work, we have focused on the synthesis of 1,3-oxazine derivatives by three-component coupling of $\alpha$, or $\beta$ naphthol, formaldehyde, and anilines in presence of catalytic amount of phosphoric acid using water as solvent at room temperature.

\section{Experimental}

\subsection{Apparatus and Analysis}

The melting points of the compounds were determined are uncorrected. The synthesized products were monitored on silica gel $G$ plates by using pet ether: ethyl acetate (8:2) as mobile phase. FT-IR spectra were recorded on a Shimadzu Miracle 10 ATR spectrometer. ${ }^{1} \mathrm{H}$ NMR spectra were recorded on a Bruker $500 \mathrm{MHz}$ spectrometer with $\mathrm{CDCl}_{3}$ as the solvent and TMS as the internal reference. ${ }^{13} \mathrm{C}$ NMR spectra were recorded on Bruker $125 \mathrm{MHz}$ spectrometer with $\mathrm{CDCl}_{3}$ as the solvent. All compounds are purified by column chromatography using silica gel (70-230 mesh) and solventspet-ether: ethyl acetate as 8:2. Elemental analysis carried out using CHN elemental analyzer. 
2.2. General Experimental procedure:

To a mixture of $\alpha$ or $\beta$-naphthol $(1.0 \mathrm{mmol})$, formaldehyde ( $2.0 \mathrm{mmol}), 1 \mathrm{ml}$ polyphosphoric acid $(85 \%)$ and $(1.0 \mathrm{mmol})$ aniline, $5 \mathrm{ml}$ water was added . The reaction mixture was stirred for appropriate time at room temperature .The progress of reaction was monitored on TLC .After completion of reaction, the reaction mixture was extracted with $5 \mathrm{ml}$ diethylether.The organic layer was washed with brine solution and dried over anhydrous sodium sulfate .The crude product was purified by using silica gel column chromatography (20\% ethylacetate in light petroleum) to get pure white solid product with excellent yields.

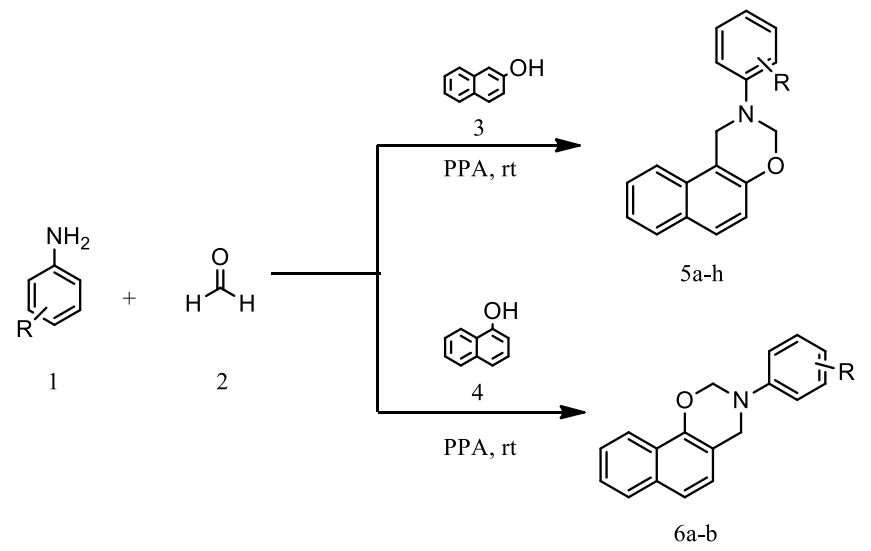

Scheme-I: PPA catalyzed synthesis of 1, 3 Oxazine derivatives in water

\section{Spectral data:}

5a 2-phenyl-2,3-dihydro-1H-naphtho[1,2-e] [1,3] oxazine

IR $\left(\mathrm{KBr}, \mathrm{cm}^{-1}\right) 2920,1615,1580,1460,1210,1036 ;{ }^{1} \mathrm{H}-$ NMR (200MHz, DMSO-d6)d = 5.07 (s,2H, Ar-CH2N) ,6.05 (s,2H, N-CH2-O) ,6.94-8.09(11H, Ar-H) ; ${ }^{13} \mathrm{C}$ NMR( 200MHz,DMSO) 57.4,93.2 ,111.2,114.2, 118.0,120.8,121.7,123.4,126.3,128.2,128.3,128.5,129.4, 131.4,149.6,151.8; (ESI):m/z $261.33(\mathrm{~m}+1)$ :Elemental analysis $\mathrm{C}_{18} \mathrm{H}_{15} \mathrm{NO}$ Calculated: C 82.73\% H 5.79\% N 5.36\% O 6.12\% Found C 82.70\% H 5.71\% N $5.32 \%$ O $6.05 \%$

5b 2-(2-methylphenyl)-2,3-dihydro-1H-naphtho[1,2e] $[1,3]$ oxazine
IR $\left(\mathrm{KBr}, \mathrm{cm}^{-1}\right)$ 2922, 1613,1576,1456,1202,996; ${ }^{1} \mathrm{H}-$

NMR (200MHz, DMSO-d6)d =2.10(s,3H,Ar-CH3 $) 5.03$ (s,2H, Ar- $\left.\mathrm{CH}_{2}-\mathrm{N}\right)$, , 6.8(s,2H, N-CH2-O) ,5.02-8.12 (m,10H, Ar-H); ${ }^{13} \mathrm{C}$ NMR (200MHz,DMSO) 17.8,58.2 ,93.1,110.3,111.2,118.2,120.4,123.0,123.1,12 6.1,126.4, 128.2,128.4,128.6,131.2, 131.5,132.7,147.1,151.5 ; MS(ESI):m/z 275.35

$(\mathrm{m}+1)$ :Elemental analysis $\mathrm{C}_{19} \mathrm{H}_{17} \mathrm{NO}$ Calculated: $\mathrm{C}$ 82.88\% H 6.22\% N 5.09\% O 5.81\% Found C 82.79\% $\mathrm{H} 6.24 \%$ N 5.11\% O 5.80\%

5c 2-(4-methylphenyl)-2,3-dihydro-1H-naphtho[1,2e] $[1,3]$ oxazine

IR $\left(\mathrm{KBr}, \mathrm{cm}^{-1}\right) 2924,1610,1573,1452,1200,992 ;{ }^{1} \mathrm{H}-$ NMR (200MHz, DMSO-d 6 ) d =2.34(s,3H Ar-CH3 ), $5.03\left(\mathrm{~s}, 2 \mathrm{H}, \mathrm{Ar}-\mathrm{CH}_{2}-\mathrm{N}\right), 6.08\left(\mathrm{~s}, 2 \mathrm{H}, \mathrm{N}-\mathrm{CH}_{2}-\mathrm{O}\right), 6.62-$ 8.09 (m,10H, Ar-H); ${ }^{13} \mathrm{C}$ NMR (200MHz,DMSO) 21.1,57.5, 93.2,111.3,112.5,112.6,118.2,120.6,123.6,126.1,128.1,1 28.2,128.6, 129.6,129.7, 130.5, 131.5,146.3,151.5 ;MS(ESI):m/z 275.35

$(\mathrm{m}+1)$ :Elemental analysis $\mathrm{C}_{19} \mathrm{H}_{17} \mathrm{NO}$ Calculated: $\mathrm{C}$ 82.88\% H 6.22\% N 5.09\% O 5.81\% Found C 82.80\% H 6.21\% N 5.05\% O 5.83\%

5d 2-(3-nitrophenyl)-2,3-dihydro-1H-naphtho[1,2-e] $[1,3]$ oxazine

IR $\left(\mathrm{KBr}, \mathrm{cm}^{-1}\right)$ 2926, 1620,1580,1465,1365,1220,1012; ${ }^{1} \mathrm{H}-\mathrm{NMR}$ (200MHz, DMSO-d6) d = 5.03 (s,2H, Ar$\left.\mathrm{CH}_{2}-\mathrm{N}\right), 6.05\left(\mathrm{~s}, 2 \mathrm{H}, \mathrm{N}-\mathrm{CH}_{2}-\mathrm{O}\right), 6.90-8.13$ (m,10H, Ar$\mathrm{H}) ;{ }^{13} \mathrm{C}$ NMR (200MHz, DMSO) 57.9,93.2,107.4, 11.7, 113.2,113.3,118.4,120.6,120.7,123.2,126.1,128.1, 128.4,128.6 ,130.7, 131.9, 148.6,150.7,151.9 ; MS(ESI):m/z $306.32(\mathrm{~m}+1)$ :Elemental analysis $\mathrm{C}_{18} \mathrm{H}_{14} \mathrm{~N}_{2} \mathrm{O}_{3}$ Calculated: $\mathrm{C} 70.58 \% \quad \mathrm{H} 4.61 \% \quad \mathrm{~N} 9.15 \%$ O $15.67 \%$ Found $70.60 \%$ H $4.55 \%$ N 9.10\% O $15.60 \%$

5e 2-(3-methylphenyl)-2,3-dihydro-1H-naphtho[1,2e] $[1,3]$ oxazine

IR $\left(\mathrm{KBr}, \mathrm{cm}^{-1}\right) 2925,1615,1578,1459,1206,1001 ;{ }^{1} \mathrm{H}-$ NMR (200MHz, DMSO-d6) d = 5.02 (s,2H, Ar- $\mathrm{CH}_{2}-$ $\mathrm{N})$, , 6.09(s,2H, N-CH $2-\mathrm{O}), 6.6-8.13(\mathrm{~m}, 10 \mathrm{H}, \mathrm{Ar}-$ $\mathrm{H}) ;{ }^{13} \mathrm{C}$ NMR (200MHz,DMSO) 21.3,57.5,93.2,111.1, 
111.3,

117.3,118.2,118.5,120.6,123.1,126.1,128.2,128.5,128.6, 128.7, 129.3, 131.5,149.2,151.5 ; MS(ESI):m/z 275.35 $(\mathrm{m}+1)$ :Elemental analysis $\mathrm{C}_{19} \mathrm{H}_{17} \mathrm{NO}$ Calculated: $\mathrm{C}$ 82.88\% H 6.22\% N 5.09\% O 5.81\% Found C 82.76\% 119.8,

H $6.23 \%$ N 5.10\% O 5.82\%

5f 2-(4-bromophenyl)-2,3-dihydro-1H-naphtho[1,2-e] $[1,3]$ oxazine

IR $\left(\mathrm{KBr}, \mathrm{cm}^{-1}\right) 2930,1622,1580,1460,1221,1020 ;{ }^{1} \mathrm{H}-$ NMR (200MHz, DMSO-d6) d =5.03 (s,2H, Ar- $\mathrm{CH}_{2}-$ $\mathrm{N}),, 6.05\left(\mathrm{~s}, 2 \mathrm{H}, \mathrm{N}-\mathrm{CH}_{2}-\mathrm{O}\right), 6.35-8.12(\mathrm{~m}, 10 \mathrm{H}, \mathrm{Ar}-$ $\mathrm{H}) ;{ }^{13} \mathrm{C}$ NMR (200MHz,DMSO) 21.1,57.5,93.2,112.5, 112.6,118.1,118.2,120.7,123.1,126.1,128.2,128.3,128.5, 129.7,129.9,130.5,131.4,146.5,151.3; MS (ESI):m/z $340.22(\mathrm{~m}+1)$ : Elemental analysis $\mathrm{C}_{18} \mathrm{H}_{14} \mathrm{BrNO}$ Calculated: C 63.55\% H 4.15\% Br 23.49\% N 4.12\% O $4.70 \%$ Found C $63.62 \%$ H 4.18\% Br 23.50\% N $4.10 \%$ O 4.74\% 5g 2-(4-methoxyphenyl)-2,3dihydro-1H-naphtho[1,2-e] [1,3] oxazine IR $\left(\mathrm{KBr}, \mathrm{cm}^{-1}\right) 2982,1618,1576,1461,1216,991 ;{ }^{1} \mathrm{H}-$ NMR (200MHz, DMSO-d6) d =3.81(s,3H,Ome) 5.01 (s,2H, Ar- $\left.\mathrm{CH}_{2}-\mathrm{N}\right)$, , 6.08 (s,2H, N-CH2-O) ,6.65-8.14 (m,10H, Ar-H); ${ }^{13} \mathrm{C}$ NMR (200MHz,DMSO) 55.6, 57.4, 93.2,111.2,115.1,115.4,118.37,120.6,123.2,126.4,128.1, 128.2,128.5,131.5,141.6,151.4,152.6 ; MS(ESI):m/z $291.35(\mathrm{~m}+1)$ :Elemental analysis $\mathrm{C}_{19} \mathrm{H}_{17} \mathrm{NO}_{2}$

Calculated: C 78.33\% $\quad$ H 5.88\% N 4.81\% O 10.98\% Found C 78.32\% $\quad$ H 5.90\% N 4.83\% O 10.96\% 5h 2-(2-nitrophenyl)-2,3-dihydro-1H-naphtho[1,2-e] $[1,3]$ oxazine

IR $\left(\mathrm{KBr}, \mathrm{cm}^{-1}\right)$ 2924,1620,1570,1451,1360,1209,1012; ${ }^{1} \mathrm{H}-\mathrm{NMR}\left(200 \mathrm{MHz}, \mathrm{DMSO}-\mathrm{d}_{6}\right) \mathrm{d}=5.01(\mathrm{~s}, 2 \mathrm{H}, \mathrm{Ar}-$ $\left.\mathrm{CH}_{2}-\mathrm{N}\right), 6.06\left(\mathrm{~s}, 2 \mathrm{H}, \mathrm{N}-\mathrm{CH}_{2}-\mathrm{O}\right), 6.91-8.13$ (m,10H, Ar$\mathrm{H}) ;{ }^{13} \mathrm{C}$ NMR (200MHz,DMSO) 56.9,92.2, 110.5, 111.7, 118.6,119.4,120.4,123.2,126.1,126.3,128.1,128.2, 128.6,131.9, 135.2,140.2, 143.3,151.9 ;MS (ESI):m/z $306.32(\mathrm{~m}+1)$ :Elemental analysis $\mathrm{C}_{18} \mathrm{H}_{14} \mathrm{~N}_{2} \mathrm{O}_{3}$ Calculated: C 70.58\% H 4.61\% N 9.15\% O 15.67\% Found 70.61\% H 4.57\% N 9.12\% O 15.62\% 6a 3-phenyl-3,4-dihydro-2H-naphtho[2,1-e] [1,3] oxazine
IR $\left(\mathrm{KBr}, \mathrm{cm}^{-1}\right) 2920,1614,1577,1457,1204,998 ;{ }^{1} \mathrm{H}-$

NMR (200MHz, DMSO-d6) d = 5.01 (s,2H, Ar- $\mathrm{CH}_{2}-$ $\mathrm{N}), 6.02\left(\mathrm{~s}, 2 \mathrm{H}, \mathrm{N}-\mathrm{CH}_{2}-\mathrm{O}\right)$, 6.91-8.13 (m,10H, Ar-H); ${ }^{13} \mathrm{C}$ NMR (200MHz,DMSO) 59.7, 93.2, 113.2, 114.1, 121.7,122.7,124.8,125.2,125.4,125.7,127.3,129.4,132.7, 149.2,149.4; MS(ESI):m/z $261.33(\mathrm{~m}+1):$ Elemental analysis $\mathrm{C}_{18} \mathrm{H}_{15} \mathrm{NO}$ Calculated: C 82.73\% H 5.79\% N 5.36\% O 6.12\% Found 82.61\% H 5.87\% N 5.40\% O $6.12 \%$

6b 3-(4-methoxyphenyl)-3,4-dihydro-2Hnaphtho[2,1-e] [1,3] oxazine IR $\left(\mathrm{KBr}, \mathrm{cm}^{-1}\right)$ 2930,1615,1572,1462,1214,996; ${ }^{1} \mathrm{H}-$ NMR (200MHz, DMSO-d6) d = 5.01 (s,2H, Ar- $\mathrm{CH}_{2}-$ N) ,6.02(s,2H, N-CH2-O) ,6.91-8.13 (m,10H, Ar-H); ${ }^{13} \mathrm{C}$ NMR (200MHz,DMSO) 59.7,92.2, 113.2, 114.2, 119.4,121.7,122.7,124.4,125.4,125.7,127.3,129.4,132.2, 149.2,149.4; MS(ESI):m/z $291.35(\mathrm{~m}+1)$ :Elemental analysis $\mathrm{C}_{19} \mathrm{H}_{17} \mathrm{NO}_{2}$ Calculated: C 78.33\% $\quad \mathrm{H} 5.88 \%$ N 4.81\% O 10.98\% Found 78.34\% H 5.89\% N $4.82 \%$ O $10.99 \%$

\section{RESULTS AND DISCUSSION}

The effect of reaction conditions on the formation of 1, 3-oxazine catalyzed by PPA in the presence of water was summarized in Table-1.

Table-1: Screening of solvent for synthesis of compound $\mathbf{5 a}$ in different solvent.

\begin{tabular}{cccc}
\hline Entry & Solvent & $\begin{array}{c}\text { Time } \\
(\text { min.) }\end{array}$ & Yield (\%) \\
\hline 1 & Solvent free & 240 & 25 \\
2 & $\mathrm{H}_{2} \mathrm{O}$ & 16 & 95 \\
3 & $\mathrm{EtOH}$ & 20 & 75 \\
4 & $\mathrm{MeOH}$ & 20 & 72 \\
5 & $\mathrm{DMF}$ & 20 & 32 \\
6 & $\mathrm{DMSO}$ & 20 & 35 \\
7 & $\mathrm{DCM}$ & 20 & 49 \\
8 & $\mathrm{CH}_{3} \mathrm{CN}$ & 20 & 35 \\
\hline
\end{tabular}


We have studied the three component reaction of $\alpha$ or $\beta$-naphthol, aniline and formaldehyde (1:1:2) using PPA $(1 \mathrm{ml})$ in absence of water at room temperature for $240 \mathrm{~min}$. to obtained 25\% 1, 3 oxazine derivative $5 \mathrm{a}$ (entry-1). In the presence solvent such as $\mathrm{EtOH}$, $\mathrm{MeOH}, \mathrm{DMF}, \mathrm{DMSO}, \mathrm{DCM}$ and $\mathrm{CH}_{3} \mathrm{CN}$ poor yield was obtained (entries3-8). No product was formed, when the mixture was stirred under similar reaction conditions in the absence of PPA, even after prolonged stirring. But in the presence of water and 1 $\mathrm{ml}$. of catalyst reaction proceeded rapidly and excellent yield of product was obtained.

Aromatic amines bearing electron donating substituents such as $-\mathrm{Me}$, -Ome gave good yields in less time, while in the presence of electron withdrawing groups reaction required more time and poor yield. In this three component condensation, using $\beta$-naphthol reaction proceeded at faster rate, while in the presence of $\alpha$-naphthol, rate of reaction was decreased, also affected the total yield of the product. Aliphatic amines gave the side products and hence required product could not be isolated.The electrophilic substitution reaction of $\beta$-naphthol is always more reactive than $\alpha$-naphthol because $\beta$ naphthol gives more resonating structures than $\alpha$ naphthol. Due to their stabilization $\beta$-naphthol undergo faster reaction rate than $\alpha$-naphthol.

The use of water as an universal solvent has certain advantages like low cost, easy availability in abundantquantity, non-inflammability, nontoxic, eco-friendly and easy product isolation, when product is solid.

Table-2: Synthesis of 1, 3 oxazine derivatives catalyzed by PPA in water at room temperature.

Entry


$5 e$<smiles>Cc1cccc(N)c1</smiles>

$5 f$<smiles>Nc1ccc(Br)cc1</smiles>

$5 g$<smiles>COc1ccc(N)cc1</smiles>

$5 \mathrm{~h}$<smiles>Nc1ccccc1[N+](=O)[O-]</smiles>

$6 a$<smiles>Nc1ccccc1</smiles>

$6 b$<smiles>COc1ccc(N)cc1</smiles><smiles>Cc1cccc(N2COc3ccc4ccccc4c3C2)c1</smiles>

17

94

74-76 [42]

16

93

$116-118$ [42]

$111-113$ [47c]

$110-112$ [42]
15

19

93

23

85

20

92

74-76 [42]<smiles>c1ccc(N2COc3c(ccc4ccccc34)C2)cc1</smiles><smiles>COc1ccc(N2COc3c(ccc4ccccc34)C2)cc1</smiles>

86

300 [42]

\section{IV.CONCLUSION}

\section{ACKNOWLEDGMENTS}

In summary, we have reported a simple and ecofriendly protocol for the synthesis of a variety of 1,3- The authors gratefully acknowledge the Central oxazine derivatives via one-pot three-component Instrumental Facility, Savitribai Phule,Pune condensation of $\alpha$, or $\beta$ - naphthol anilines, University, Pune, India and DST - FIST sponsored formaldehyde catalyzed by polyphosphoric acid Central Instrumentation Laboratory, Dada Patil catalyst in water at room temperature. This method Mahavidyalaya, Karjat.Dist- Ahmednagar, India for offers a good supplement method to synthesize 1,3- the spectral analysis and financial support. oxazine derivatives at low cast, using safer catalysts, easy workup, short reaction time, higheryields. 


\section{REFERENCES}

[1]. P. Laszlo, Organic Reaction: Simplicity and Logic, Wiley New York, (1995).

[2]. T. Tanaka, F. Toda Solvent-free Organic Synthesis, Chem, Rev.100, 1025-1074, (2000).

[3]. M. Alam, R. Varala, S.R. AdapaSynlett, 67-70, (2003).

[4]. A. Dondi, A. Massi, E. Minghini, S. Sabbatini, V. Bertoasi J. Org. Chem,68, 6172-6183, (2003).

[5]. R. Hanselmann, J. C. Zhou, P. Ma, P. N. Confalone J. Org. Chem,68, 8739-8741, (2003).

[6]. R. S. Thombal, V. H.Jadhav J. Chem. Appl. Biochem., 2,111 (2015).

[7]. R. S.Thombal, Y. R. Lee, Org. Lett. 2018, 20, 4681.

[8]. B. Ganem, Acc. Chem. Res., 42, 463, (2009).

[9]. A. Domling, Chem. Rev., 106, 17, (2006).

[10]. A. I. Domling, Ugi Angew, Chem. Int. Ed., 39, 3168, (2000).

[11]. V.Nair, C. Rajesh, A.U. Vinod, S. Bindu, A.Sreekanth, J. Mathen, A. Balagopal, Acc. Chem. Rev., 36, 899, (2003).

[12]. S. L. Schreiber Science,287, 1964, (2000).

[13]. L.A. Thompson, Curr. Opin. Chem. Biol., 4, 324, (2000).

[14]. B. M. Trost, Angew. Chem. Int. Ed. Engl., 34, 259, (1995).

[15]. L.F. Tietze Chem. Rev., 96, 115, (1996).

[16]. B. M. Trost, Science,254, 1471, (1991).

[17]. M. S. Singh, G. C. Nandi, S. Samai Green Chem.,14, 447, (2012).

[18]. L. R. Wen, Z. R. Li, H. Cao. Green Chem., 14, 707, (2012).

[19]. T. Kurz Tetrahedron, 61, 3091, (2005).

[20]. A.I. Meyers, G. R. Malone, J. Org. Chem., 39, 618, (1974).

[21]. A. I. Meyers, E. M. Smith J. Org. Chem., 37, 4289, (1972).

[22]. M. Adib, E. Sheibani, K. Mostofi, K. Ghanbary, H. R. Bijanzadeh Tetrahedron, 62, 3435, (2006).
[23]. N. Kajino, Y. Shibouta, K. Nishikawa, K. Meguro, Chem. Pharm. Bull., 11, 2896, (1991).

[24]. Y. Katsura, S. Nishino, H. Takasugi, Chem. Pharm. Bull., 11, 2937, (1991).

[25]. B. O. Buckman, R. Mohan, S. Koovakkat, Bioorg. Med. Chem. Lett., 8, 2235, (1998).

[26]. H. V. Poel, G. Guilaumet, Viaud-Massuard, Tetrahedron Lett., 43, 1205, (2002).

[27]. G. Mehdi, O. Abolfazl, R. M. Synth. Commun., 38, 4125, (2008).

[28]. P. Zhang, E. A. Terefenko, A. Fensome, J. Wrobel, R. Winneker, Z. Zhang, Bioorg. Med. Chem. Lett., 13, 1313, (2003).

[29]. F. A. Kerdesky, Tetrahedron Lett., 46, 1711, (2005).

[30]. B. S. Holla, K. N. Poojary, B. S. Rao, M. K. Shivandana Eur. J. Med. Chem., 37, 511, (2002).

[31]. G. Martin, R. A. Lahti, A.D. Rudzik, D. J. Duchamp, C. Chidester, T. J. Scahill,Med. Chem., 21, 542, (1978).

[32]. G. Thomas, D. V. Mehta, R. Tahilramani, D. Joy, P. K. Talwalker, J. Med. Chem., 14, 335, (1971).

[33]. B. S. Holla, K. N. Poojary, B. Kalluraya, P. V. Gowda II Farmaco, 51, 793, (1996).

[34]. S. F. Wnuk, Tetrahedron, 49, 9877, (1993).

[35]. A. A. Yuzhakov, Z. G. Chidgeavadze, R. S. Beabealashvilli FEBS, 306, 185, (1992).

[36]. A. A. Yuzhakov, Z. G. Chidgeavadze, R. S. Beabealashvilli, A. A. Kraevskii, G. A. Galegov, M. N. Kornecva, D. N. Nosik, T. Y. Kilesso, Bioorg. Khim., 17, 504, (1991).

[37]. L. Le Hir de Fallois, J. L. Decout, M. Fontecare, J. Chem. Soc. Perkin Trans, 17, 2587, (1997).

[38]. M. Tomasulo, S. Sortino, F. M. Raymo, Org. Lett., 7, 1109, (2005).

[39]. C. S. Higham, D. P. Dowling, J. L. Shaw, A. Cetin, C. J. Ziegler and J. R. Farrell, Tetrahedron Lett., 47, 4419, (2006).

[40]. W. J. Burke, E. L. M. Glennie and C. Weatherbee, J. Org. Chem., 49, 909, (1964). 
[41]. P. M. Bijoy, K. Awanit, S. Satyasheel, P. K. Shukla and N. Mahendra, Eur. J. Med. Chem., 45, 1502, (2010).

[42]. P. M. Bijoy and N. Mahendra, J. Heterocyclic Chem., 46, 1003, (2009).

[43]. B. S. Suryakant, F. S. Kiran, B. S. Bapurao and S. S. Murlidhar, J. Koran Chem. Soci., 54, 437, (2010).

[44]. I. Szatmari, F. Fulop,Curr. Org. Synth., 1, 155, (2004).

[45]. I. Szatmari, F. Fulop, Tetrahedron, 69, 1255, (2013).

[46]. R. Csutortoki, I. Szatmari, A. Koch, M. Heydenreich, I. Starke, F. Fulop, E. Kleinpeter, Tetrahedron, 68, 6284, (2012).

[47]. S. A. Sadaphal, S. S. Sonar, K. F. Shelke, B. B. Shingate, M. S. Shingare Org. Commun., 3, 1, (2010).

[48]. A. H. Kategaonkar, S. S. Sonar, B. B. Singate, M.

S. Shingare, Green Chem. Lett. Rev., 3, 213, (2010).

[49]. Z. Turgut, E. Pelit, A. Koycu, Molecules, 12, 345, (2007).

[50]. H. Cao. H. F. Jiang, C. R. Qi, W. J. Yao, H. J. Chen, Tetrahedron Lett., 50, 1209, (2009).

[51]. V. D. Dhakane etal, C. R. Chimie, 17, 431-436, (2014).

[52]. F. Torav, G. Ochoa, Org. Lett., 2, 965, (2000).

[53]. J. E. Kang, H. B. Kim, J. W. Lee, S. Shin, Org Lett., 8, 3537, (2006).

[54]. M. Lei, L. Ma, I. Hu, Tetrahedron Lett., 50, 6393, (2009).

[55]. Comprehensive Organic Synthesis Vol.2, 707731, (1991).

\section{Cite this article as :}

Santosh R. Kshirsagar, Ashok S. Pise, Sagar I. Shinde, Sandip P. Gondake, Valmik S. Kapase, "One-Pot Green Method for the Synthesis of Oxazine Derivatives Under Aqueous Medium", International Journal of Scientific Research in Science and Technology (IJSRST), Online ISSN : 2395-602X, Print ISSN : 2395-6011, Volume 7 Issue 4, pp. 332-339, July-August 2020. Available at doi $\quad$ : https://doi.org/10.32628/IJSRST207482 Journal URL : http://ijsrst.com/IJSRST207482 